\title{
The Central Role of Systematics in Marine BIODIVERSITY PROBLEMS
}

By Michael Vecchione and Bruce B. Collette

$\mathrm{T}_{\mathrm{H}}$ 1 he COnTributions in this special issue clearly demonstrate the broad range of problems involved in the subject of marine biological diversity. One central theme, however, is the need for accurate identification of the organisms being studied. Whether questions investigated involve ecosystem function or the extent of mixing in a gene pool, it is essential first to know what lives where. This requirement has recently been voiced eloquently by the systematics community (Systematics Agenda 2000, 1994) in a volume that also summarizes the components of the science of systematics, such as alpha-level taxonomy and phylogenetic analysis. The importance of systematics and taxonomy has been highlighted by a National Academy of Sciences panel report on marine biodiversity (National Academy of Sciences, 1995). A thorough review and proposed world strategy for understanding and preserving marine biodiversity (Norse, 1993) also emphasized the need for good taxonomic knowledge and the scientific community's increasing difficulties in fulfilling that need.

A consensus has developed among people working on biodiversity indicating that there are three basic levels of biological diversity. These are 1) diversity among ecosystems, including landscapescale questions; 2) phylogenetic diversity, which includes diversity of species as well as their evolutionary uniqueness and relationships among them; and 3 ) genetic diversity within each species. Effective research at all three levels requires the ability to distinguish among species. For example, understanding of ecosystem diversity is based on determining the struc-

M. Vecchione and B.B. Collette, NOAA, National Marine Fisheries Service, National Systematics Laboratory, National Museum of Natural History, Washington, DC 20560, USA.
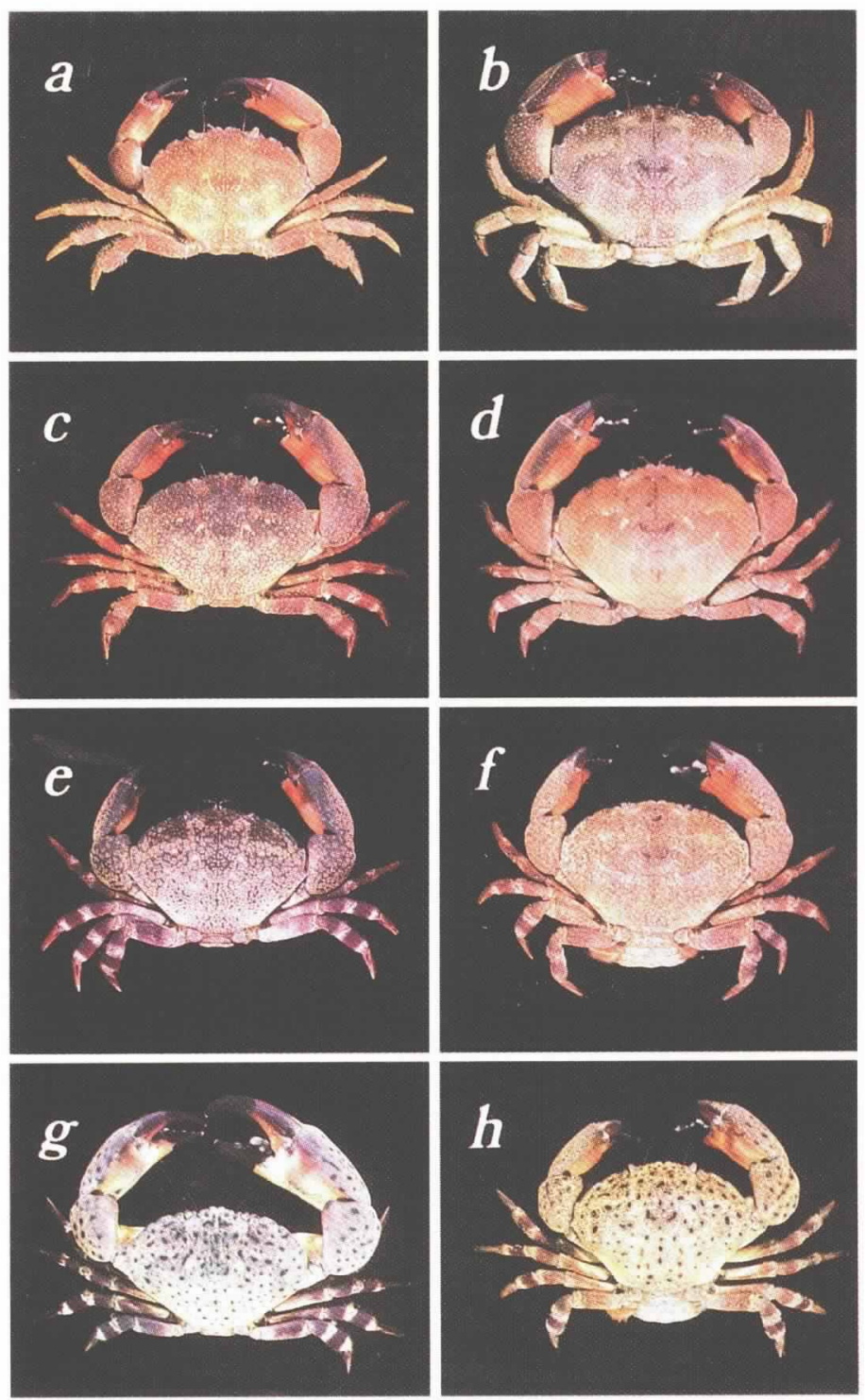

Fig. 1: Detailed morphological and biochemical examination of marine invertebrates has revealed that many groups once thought to comprise a single species actually are assemblages of morphologically similar species. This is true even for taxa that are large and economically or ecologically important. For example, Williams and Felder (1986) showed that stone crabs from the Gulf of Mexico previously assigned to Menippe mercenaria (Say, 1818) could be separated into two distinct species with hybrids occurring in the zone of distributional overlap. Crabs $\boldsymbol{a}$ and $\boldsymbol{b}$ are M. adina (Williams and Felder, 1986) from Louisiana. Crabs $\boldsymbol{g}$ and $\boldsymbol{h}$ are M. mercenaria from Florida. Crabs $\boldsymbol{c}-\boldsymbol{h}$ are hybrids. 
ture of biological communities and how they function. The first step toward this goal is inventory of the organisms living in the target ecosystems. which requires being able to tell one species from another. Determination of how organisms function in an ecosystem is based largely on the comparative method and thus requires an understanding of their evolutionary relationships and how evolution may have affected adaptations (Vecchione, 1994). The study of phylogenetic diversity involves broad-scale comparisons of taxa so that previously unrecognized taxa (species, genera, families, etc.) can be identified and described and the relationships among taxa can be determined (Fig. 1). To study genetic diversity, it is necessary to identify the organisms on which one is working. Such genetic studies often begin with morphological clues derived from taxonomic studies.

The National Oceanic Atmospheric Administration. NMFS, and their predecessor agencies have long recognized the need for systematic and taxonomic studies. In a recent paper, we reviewed important interactions between systematics and fisheries (Collette and Vecchione, 1995). Studies at the National Systematics Laboratory (Vecchione and Collette, 1994) have traditionally emphasized species with economic or ecological importance in U.S. waters. During this laboratory's first 50 years
(1942-1992), these studies, which by necessity are based on worldwide comparisons, resulted in the discovery of 396 new taxa of tishes, crustaceans, and mollusks (National Systematics Laboratory, 1993).

It is obvious, however, that the federal government alone cannot accomplish the systematic research and management of biological collections necessary to support the expanding requirements for understanding and protecting marine biological diversity. Broad collaboration in systematics among federal and state agencies, museums, and universities must be continued and expanded (Vecchione and Collette, 1996). Unfortunately, the number of taxonomic specialists is decreasing. as is the amount of support for natural history collections (Poss and Collette. 1995).

The seas comprise $>70 \%$ of the earth's surface and $99 \%$ of the volume of the biosphere, but the marine environment remains largely unexplored. Although the seas hold the greatest variety of basic body types (phyla), estimates of marine diversity vary greatly. This is largely because of the rudimentary state of our knowledge about marine organisms in comparison with terrestrial animals and plants. To rectify this situation, it is imperative that we repair our systematics infrastructure. Only then can we make rapid progress in the funda- mental task of distinguishing one species from another and thereby solidify the foundation of systematic knowledge upon which understanding of biodiversity depends.

\section{References}

Collette, B.B. and M. Vecchione. 1995: Interaction between fisheries and systematics. Fisheric's, 20, 20-25.

National Academy of Sciences, 1995: Linderstanding Marine Biodiversity: A Research Agendes for the Nation. National Academy Press. Washington, DC, $11+\mathrm{pp}$.

National Systematics Laboratory, 1993: Fifty year bibliography (1942-1992) of the National Systematics laboratory. National Systematics Laboratory. $26 \mathrm{pp}$.

Norse, E.A. 1993: Global Marine Biodiversity. 1sland Press, Washington. DC. $383 \mathrm{pp}$.

Poss. S.G. and B.B. Collette, 1995: Second survey of fish collections in the United States and Canada. Copeia, 1995, 48-70).

Systematics Agenda 2000, 1994: Systematics Agenda 2000: charting the biosphere. Technical report. Systematics Agenda 20000. 34 pp.

Vecchione, M., 1994: Systematics and the lifestyle and performance of cephalopods. Mur. Behar. Physiol., 25, 179-191. and B.B. Collette. 1994: Profile: National Systematics Laboratory. Fisheries, 19, 26. and B.B. Collette, 1996: Fisheries angencies and marine biodiversity. Ann. Missouri Bot. Gard. 83, 29-36.

Williams. A.B. and D.L. Felder, 1986: Analysis of stone crabs, Menippe mercenaria (Say), restricted, and a previously unrecognized speces dexcribed (Decapoda: Xanthidae) Proc. Biol. Soc. Wash., 99, 517-543. 口 